\title{
A combined risk model for the multi- encompassing identification of heterogeneities of prognoses, biological pathway variations and immune states for sepsis patients
}

Zong-xiu Yin ${ }^{1,2}$, Chun-yan Xing ${ }^{1,2}$, Guan-hua Li ${ }^{1,2}$, Long-bin Pang ${ }^{1,2}$, Jing Wang ${ }^{1,2}$, Jing Pan ${ }^{1,2}$, Rui Zang ${ }^{1,2}$ and Shi Zhang ${ }^{1,2^{*}}$

\begin{abstract}
Background: Sepsis is a highly heterogeneous syndrome with stratified severity levels and immune states. Even in patients with similar clinical appearances, the underlying signal transduction pathways are significantly different. To identify the heterogeneities of sepsis from multiple angles, we aimed to establish a combined risk model including the molecular risk score for rapid mortality prediction, pathway risk score for the identification of biological pathway variations, and immunity risk score for guidance with immune-modulation therapy.
\end{abstract}

Methods: We systematically searched and screened the mRNA expression profiles of patients with sepsis in the Gene Expression Omnibus public database. The screened datasets were divided into a training cohort and a validation cohort. In the training cohort, authentic prognostic predictor characteristics (differentially expressed mRNAs, pathway activity variations and immune cells) were screened for model construction through bioinformatics analysis and univariate Cox regression, and a $P$ value less than 0.05 of univariate Cox regression on 28 -day mortality was set as the cut-off value. The combined risk model was finally established by the decision tree algorithm. In the validation cohort, the model performance was assessed and validated by $C$ statistics and the area under the receiver operating characteristic curve (AUC). Additionally, the current models were further compared in clinical value with traditional indicators, including procalcitonin (PCT) and interleukin-8 (IL-8).

Results: Datasets from two sepsis cohort studies with a total of 585 consecutive sepsis patients admitted to two intensive care units were downloaded as the training cohort $(n=479)$ and external validation cohort $(n=106)$. In the training cohort, 15 molecules, 20 pathways and 4 immune cells were eventually enrolled in model construction. These prognostic factors mainly reflected hypoxia, cellular injury, metabolic disorders and immune dysregulation in sepsis patients. In the validation cohort, the AUCs of the molecular model, pathway model, immune model, and combined model were $0.81,0.82,0.62$ and 0.873 , respectively. The AUCs of the traditional biomarkers (PCT and IL-8) were 0.565 and 0.585 , respectively. The survival analysis indicated that patients in the high-risk group identified by models in the current study had a poor prognosis $(P<0.05)$. The above results indicated that the models in this study are all superior

\footnotetext{
*Correspondence: 394873967@qq.com

${ }^{1}$ Department of Pulmonary and Critical Care Medicine, Jinan Central Hospital Affiliated to Shandong First Medical University and Shandong Academy of Medical Sciences, Cheeloo College of Medicine, Shandong University, No. 105 Jiefang Road, Jinan 250013, Shandong Province, China

Full list of author information is available at the end of the article
} permits use, sharing, adaptation, distribution and reproduction in any medium or format, as long as you give appropriate credit to the original author(s) and the source, provide a link to the Creative Commons licence, and indicate if changes were made. The images or other third party material in this article are included in the article's Creative Commons licence, unless indicated otherwise in a credit line to the material. If material is not included in the article's Creative Commons licence and your intended use is not permitted by statutory regulation or exceeds the permitted use, you will need to obtain permission directly from the copyright holder. To view a copy of this licence, visit http://creativecommons.org/licenses/by/4.0/. The Creative Commons Public Domain Dedication waiver (http://creativeco mmons.org/publicdomain/zero/1.0/) applies to the data made available in this article, unless otherwise stated in a credit line to the data. 
to the traditional biomarkers for the predicting the prognosis of sepsis patients. Furthermore, the current study provides some therapeutic recommendations for patients with high risk scores identified by the three submodels.

Conclusions: In summary, the present study provides opportunities for bedside tests that could quantitatively and rapidly measure heterogeneous prognosis, underlying biological pathway variations and immune dysfunction in sepsis patients. Further therapeutic recommendations for patients with high risk scores could improve the therapeutic system for sepsis.

Keywords: Sepsis, Heterogeneity, Prediction model, Signal transduction pathway, Immune dysfunction

\section{Introduction}

Sepsis is a heterogeneous syndrome with an uncontrolled systemic inflammatory host response to infection which furthermore induces perpetuation of organ dysfunction [1]. Prolonged immune dysfunction induced imbalance between hyper-inflammation and anti-inflammation is identified as main reason of organ dysfunction and lethality in sepsis. Therapeutic ways for sepsis have been limited for decades and the mortality of sepsis is $10-35 \%$ $[2,3]$.

It is generally believed that previous failure of proposed treatments for septic patients is due to substantial heterogeneity in sepsis and the lack of methods to precisely classify patients at molecular biology level [4-6]. The heterogeneity in sepsis complicated the screening specific population who might benefit from adjuvant treatments. The heterogeneity of immune alterations in sepsis actually limited the efficacy and progress of immune modulation $[7,8]$. A rapid tool to quantitatively measure immune disorders could potentially classify septic population for accurate immune modulation. Therefore, Burnham et al. [9] stratified patients with sepsis to the four endotypes, and Sweeney et al. [4] built a molecular model to predict mortality for patients with sepsis according to transcriptomic data.

Nevertheless, these landmark studies for explore heterogeneity in sepsis still could not identify heterogeneity at pathogenesis level, therefore fail to achieve more-precise personalized interventions especially pathogenesisrelated therapy. This is due to that even in patients with similar clinical surfaces, underlying pathogenesis may be significant different $[10,11]$. Obviously, it is robustly believed that better insight into heterogeneity of pathogenesis is crucial to improve precise therapeutic opinions [12-14].

Under this background, we conducted a secondary analysis on high-throughput data of sepsis downloaded from a public database to construct a combined risk model including the molecular risk score, pathway risk score and immunity risk score for better insight into the heterogeneity of sepsis. The molecular risk score, as a rapid assay, could promote accuracy in sepsis prognosis for the appropriate matching of patients with resources.
The pathway risk score could indicate heterogeneity of pathogenesis for the further selection of specific adjuvant therapy. The immune risk score could provide a quantitative measure of immune dysfunction for the guidance of immune modulation therapy.

\section{Methods}

This study included the training cohort to construct the combined risk model and the validation cohort to compare the models' performance with classical septic indicators such as procalcitonin (PCT) and interleukin-8 (IL-8). The analysis flow plot is shown in Supplemental Material (SM) Fig. 1.

\section{Database search and study selection}

The data discussed in this publication were down-loaded from National Center for Biotechnology Information's (NCBI's) Gene Expression Omnibus(GEO) [15, 16]. GEO is an international public repository that archives and freely distributes microarray, next-generation sequencing, and other forms of high-throughput functional genomics data submitted by the research community $[15$, 16].

The GEO database was searched for all expression microarrays that matched terms associated with sepsis. The datasets were collected from clinical studies investigating sepsis in adults using peripheral blood within $48 \mathrm{~h}$ after ICU admission. The exclusion criteria were as follows: (1) studies with septic animal or cell models and (2) studies with a lack of prognostic information. The dataset with the maximum sample size was set as the training cohort, and another dataset was set as the external validation cohort.

All datasets were downloaded as txt files, and outputs from the mRNA array were normal-exponential background corrected and then between-array quantile normalized using the limma $\mathrm{R}$ package.

\section{Statistical analysis}

The statistical analysis included 3 steps: (1) the identification of prognostic molecules, pathways and immune cells; (2) the construction of prediction models; and (3) model evaluation. 
(1) Identification of prognostic molecules, pathways and immune cells:

For assessment underlying pathway activity variation in the sepsis samples, we utilized GSVA algorithm and canonical pathways signature, which could transform the gene by sample matrix into canonical pathways enrichment scores by sample matrix through GSVA R package $[17,18]$.

To define percentages of immune cells in the sepsis whole blood, CIBERSORT algorithm were conducted. In brief, CIBERSORT algorithm could identify and qualify the absolute fractions of 22 human immune cell phenotypes from transcriptomic data consist of diverse T cell types, B cells, NK cells, and myeloid subsets. The expressions of mRNA, pathways and immune cells were finally enrolled in prognostic features screening [19-21].

To determine prognostic signatures, the univariate Cox proportional hazards model with Bonferroni correction for multiple comparisons was performed on training cohort, with a cut-off value of $P<0.05$, using the survival R package [22-24]. Furthermore, for reducing overfitting problems, the least absolute shrinkage and selection operator (LASSO) and both backward and forward stepwise selection with the Akaike information criterion (AIC) were utilized to screen the final characteristics through the glmnet $R$ and survival $R$ packages $[25,26]$.

\section{(2) Construction of the prediction models:}

Construction of the sub-models: In the training dataset, the associations of relevant characteristics with survival were evaluated based on Cox proportional hazards regression models. Hazard ratios (HRs) were shown with their 95\% CIs. The selected characteristics were incorporated in the nomograms (risk model) to predict the probability of 28-day mortality using the rms $\mathrm{R}$ package [27, 28 . Every patient's risk score was calculated based on the predictive model, and the respective medians of the two groups were set as the cut-off value to divide the patients into high-risk and low-risk groups.

Construction of the combined-model: The risk scores, age, male sex and pneumonia status were assessed by multivariable Cox proportional hazards regression, and the variables that were significantly associated with survival were included in the combined risk model.

To simplify the model for rapid clinical application, the decision tree algorithm was utilized to further optimize the combined risk model [29].

(3) Model evaluation:
In the validation dataset, model performance was assessed according to the discrimination ability and calibration ability. Discriminating ability was evaluated using $\mathrm{C}$ statistics and the area under the receiver operating characteristic curve (AUC). Calibration of the nomograms was assessed using chi-square tests, comparing the 28-day mortality of low-risk and high-risk septic populations. In addition, the current models were further compared in clinical value with traditional indicators, including PCT and IL-8.

$\mathrm{R} \times 64$ 4.0.3 was utilized to conduct all analyses.

\section{Results \\ Patients}

After the search strategy, two mRNA datasets (GSE65682 and GSE63042) of patients with sepsis from the GEO public database (585 septic patients) were finally enrolled in the current study $[9,27]$. Sepsis was defined according to the Sepsis 1.0 criteria in these two datasets [30,31]. The patients with sepsis were all enrolled from the ICU.

The septic shock ratio in the training and validation cohorts was 34.8 and $31.1 \%$, respectively. Details of the demographic details are shown in Table 1. The GSE65682 dataset was set as the training cohort, and the GSE63042 dataset was set as the external validation cohort.

The dataset of the training cohort was uploaded by Scicluna et al. from the University Medical Center in Utrecht and the Academic Medical Center in Amsterdam. This dataset included 479 patients with sepsis and relatively complete prognostic data. In addition, 42 healthy participants (median age 35 years [IQR 30-63]; 24 [57\%] of the 42 participants were men) were also enrolled in the GSE65682 dataset.

GSE63042 data were uploaded by Langley et al. from the Immunology Department at the University of New

Table 1 Demographic and clinical characteristics

\begin{tabular}{lll}
\hline & $\begin{array}{l}\text { GSE65682 } \\
\text { Patients with sepsis: } \\
\mathbf{4 7 9}\end{array}$ & $\begin{array}{l}\text { GSE63042 } \\
\text { Patients with sepsis: } \\
\mathbf{1 0 6}\end{array}$ \\
\hline Male sex & $272(56.8 \%)$ & $63(59.4 \%)$ \\
Age & $63(18-89)$ & $59(38-85)$ \\
Country & Netherlands & USA \\
Pneumonia diagnoses & $183(38.0 \%)$ & $24(22.6 \%)$ \\
Septic shock & $167(34.8 \%)$ & $33(31.1 \%)$ \\
28day mortality & $115(24.0 \%)$ & $28(26.4 \%)$ \\
APACHE & $85(69-103)^{\text {a }}$ & $16(9-28)^{b}$ \\
Main study & Classification for sepsis & Bioinformatic analysis \\
& through transcriptomic & for host response in \\
data & sepsis \\
\hline
\end{tabular}

Definition of abbreviations: $N$ number, APACHE Acute Physiology and Chronic Health Evaluation, ${ }^{\mathrm{a}} \mathrm{APACHE}$ IV; ${ }^{\mathrm{b}} \mathrm{APACHE}$ II 


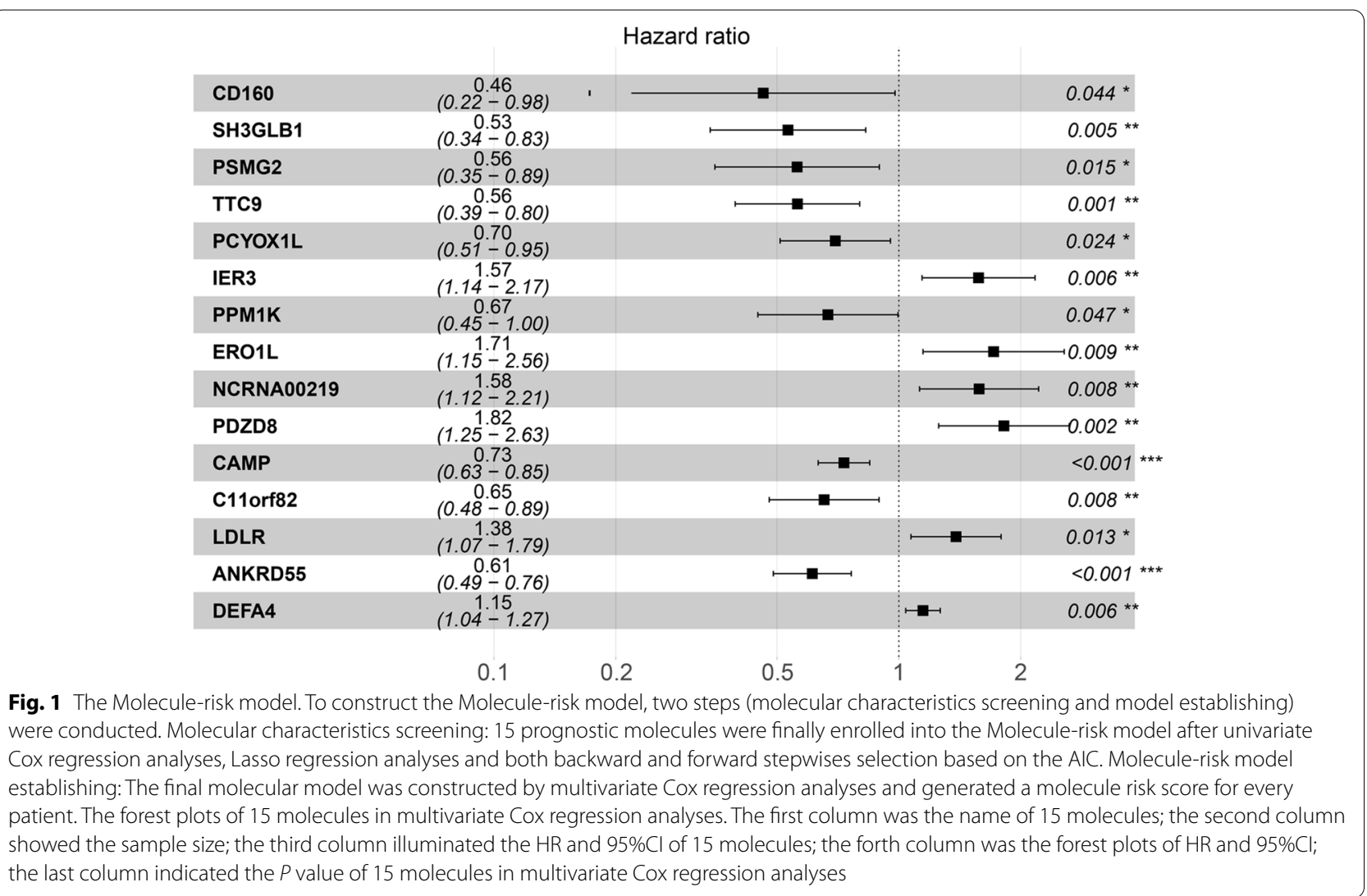

Mexico, which included 106 septic patients with 28-day mortality information.

\section{Molecular risk model}

A total of 1178 differentially expressed mRNAs among 479 patients with sepsis and 42 healthy participants were identified and screened, as shown in the volcano plot (SM Fig. 2). Furthermore, 359 molecules were further identified as prognostic molecules in sepsis and were significantly associated with 28-day cumulative mortality $(P<0.05)$, as shown in SM Table 1 . To construct the optimal model, 15 prognostic molecules were screened into the final molecular model construction. The details of the 15 molecules are shown in Fig. 1, and the screening process is shown in SM Table 2 and SM Fig. 3. The main functions of these prognostic molecules included immune response, cell apoptosis and protein synthesis in sepsis.

Based on these 15 prognostic molecules, a molecular model and nomograms were constructed, as shown in SM Fig. 4. SM Figs. 5 and 6 markedly show that patients in the high-risk group had a poor prognosis, which demonstrated that this molecular model could rapidly identify severe patients.

\section{Pathway risk model}

A total of 267 pathways that were identified through univariate Cox regression as prognostic pathways were significantly associated with 28-day cumulative mortality $(P<0.05)$, as shown in SM Table 3. Twenty pathways were further screened as final characteristics for the construction of the pathway model, as shown in Fig. 2, SM Fig. 7 and SM Table 4. The nomograms of the pathway model are shown in SM Fig. 8.

SM Figs. 9 and 10 clearly show that the number of patients who died increased with increasing pathway risk, which demonstrated that the pathway model was highly associated with the outcome of septic patients. These pathways were associated with hypoxia and immune dysregulation in sepsis patients. The network relationship of the pathways is shown in Fig. 3.

\section{Immunity risk model}

Four immune cells (naïve CD4 T cells, naïve naïve B cells, M0 macrophages and M2 macrophages) were found to be significantly associated with 28 -day cumulative mortality, as shown in Fig. 4. The nomograms of the immunity model are shown in SM Fig. 11. SM Figs. 12 and 13 clearly show that naïve $\mathrm{CD} 4 \mathrm{~T}$ cells, naïve naïve $\mathrm{B}$ cells, 


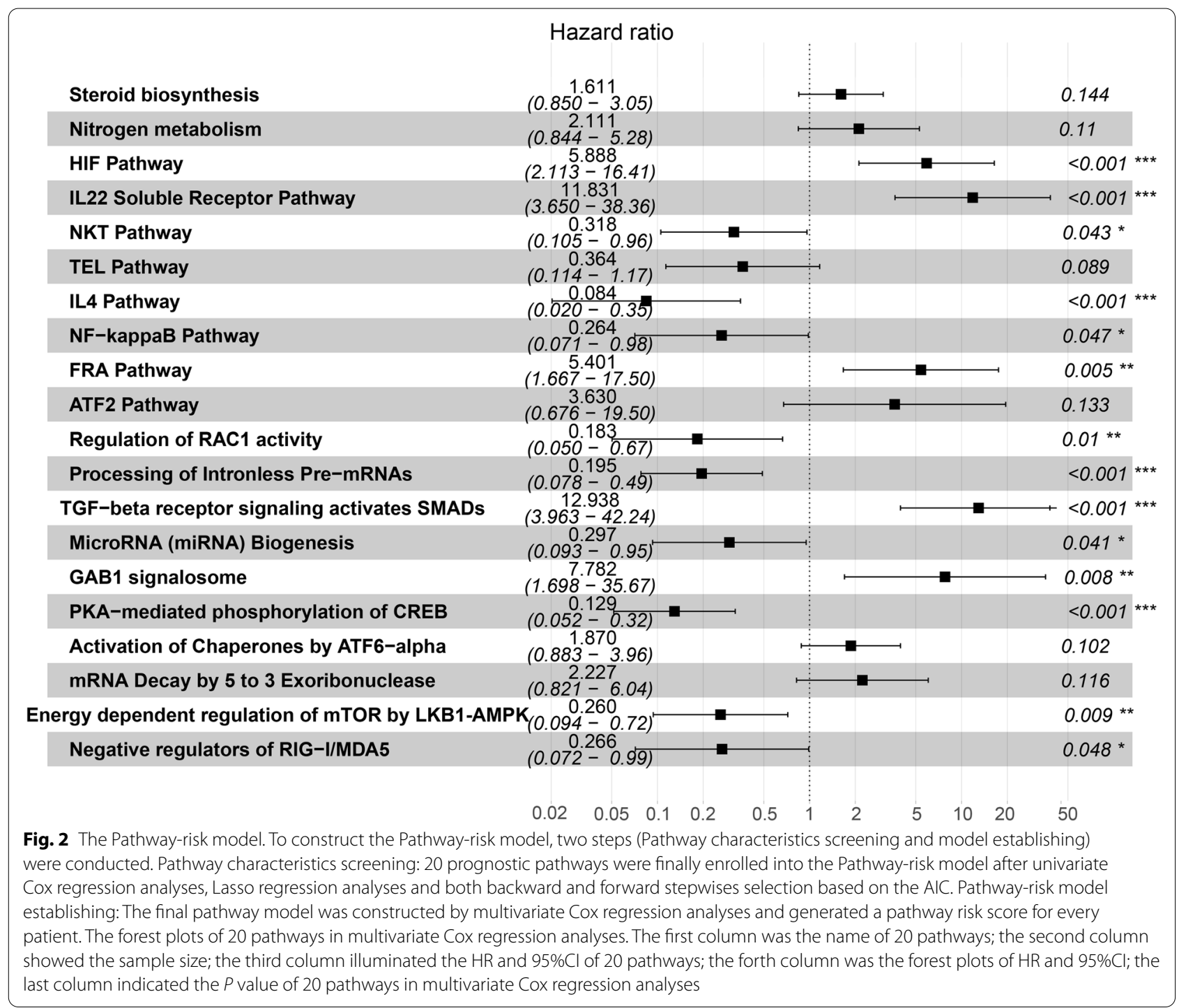

and M0 and M2 macrophages were obviously upregulated in patients in the high-risk group.

\section{Combined risk model}

To evaluate sepsis in multiple dimensions, we merged the risk factors from the molecular risk model, pathway risk model, immunity risk model and basic patient information (age, sex and pneumonia diagnoses) into the combined model. The combined risk model and nomograms were eventually built based on age, 8 prognostic molecules and 9 crucial pathways since these variables were independent prognostic factors for sepsis, as shown in SM Fig. 14.

The decision tree algorithm was utilized to further simplify and optimize the combined risk model for rapid clinical application, as shown in Fig. 5. Based on the decision tree algorithm, patients could be classified into 3 subgroups with markedly different outcomes, which could rapidly predict the outcome for septic patients.

In addition, the therapeutic recommendations for patients with high risk scores calculated by the molecular risk model, pathway risk model or immunity risk model are summarized in Table 2.

\section{Assessment of model performance}

The $\mathrm{C}$ statistics of the molecular risk model, pathway risk model, immunity risk model and combined risk model were $0.79,0.79,0.61$ and 0.873 , respectively. The AUCs were $0.81,0.82,0.62$ and 0.873 , respectively (Fig. 6).

In addition, the current models were further compared in clinical value with traditional indicators such as procalcitonin (PCT) and interleukin-8 (IL-8). The AUCs of PCT and IL- 8 were 0.565 and 0.585 , respectively, which 


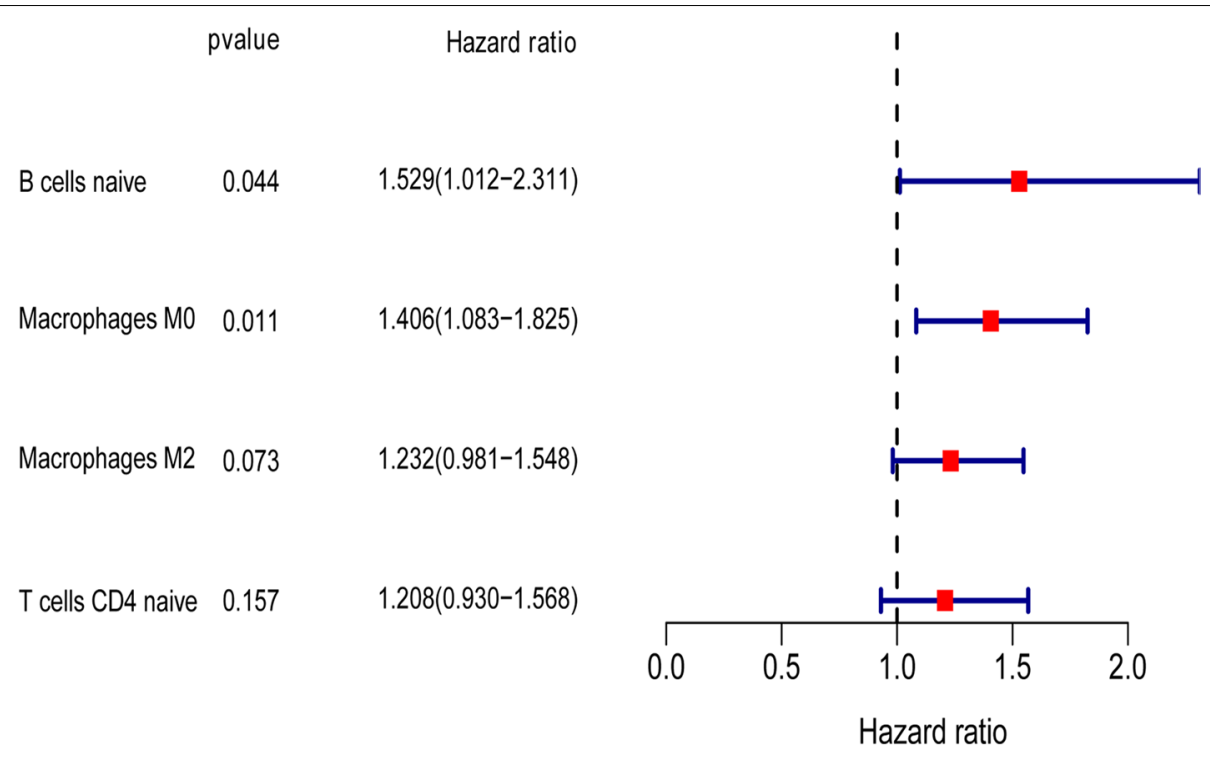

Fig. 3 Network diagram to explain the upstream and downstream relationship of the Pathway-risk model. The red pathways meant hazarded pathways ( $H R>1)$ and the blue pathways meant protective pathways $(H R<1)$. The pathways included in this model can be classified as two types, hypoxia and immune dysregulation. The pathway related to hypoxia was HIF-1 pathway. 1. Hypoxia further induced cellular injury and metabolic disorder. The pathways related to metabolic disorder included steroid biosynthesis pathway, Nitrogen metabolism pathway and energy dependent of regulation of mTOR by LKB1-AMPK. The pathways related to cellular injury included TEL pathway and ATF2 pathway. The cell repair was the negative feedback of cellular injury, and the pathways related to cellular injury included GAB1 signalosome and TGF-beta receptor signaling activates SMADs. 2. Immune dysregulation can be classified as anti-immunity, promoting-immunity and bipolar immune regulation. The pathways related to anti-immunity were TGF-beta receptor signaling activates SMADs pathway and IL22 soluble receptor pathway; the pathways related to promoting-immunity were NKT pathway, NF-kappaB pathway and PKA-mediated phosphorylation of CREB; the pathways related to bipolar immune regulation were IL-4 pathway and negative regulation of RIG-I/MDA5 pathway

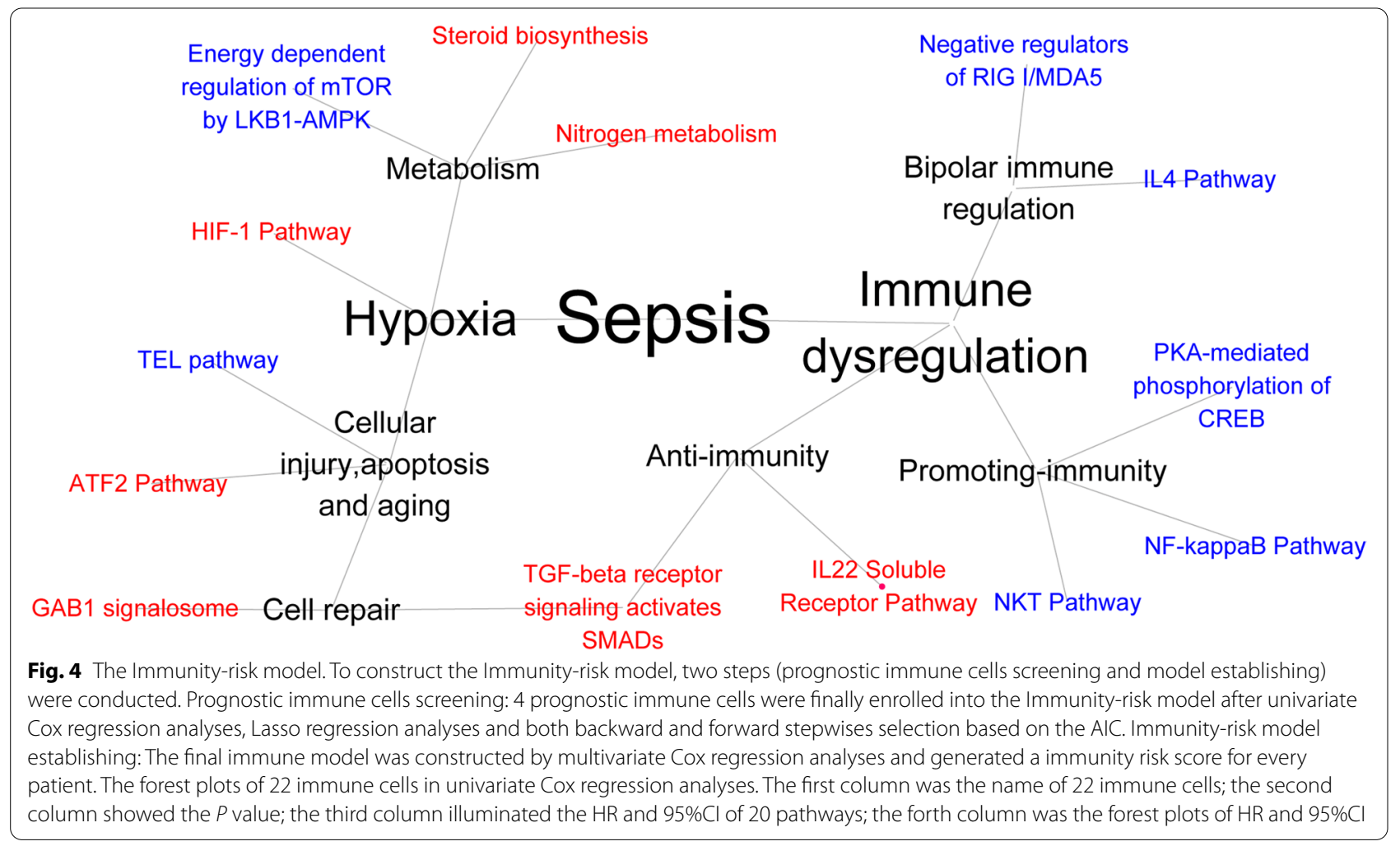


were markedly lower than those of the combined model and submodels (Fig. 6).

The above results indicated that the models in this study are all superior to traditional biomarkers for predicting the prognosis of sepsis patients.

\section{Discussion}

Sepsis identifies a highly heterogeneous population of patients, including a wide possible extent of patient conditions, complications, severity levels, pathogens, and underlying immune states $[4,5]$. To evaluate heterogeneity of sepsis in multi-dimension, the current study constructed a Combined-risk model including three sub-models (The molecule-risk model, the Pathway-risk model and the Immunity-risk model). The molecule-risk score could provide an opportunity to estimate prognosis rapidly for precise evaluation the utility of aggressive interventions. The Pathway-risk model could adequately quantify the patient's underlying pathway activity alterations for matched therapies selection. The immunity risk score from current nomograms could predict immune suppressed states based on immune cell subsets. This model could be used as the pre-hospital screening tool to identify sepsis heterogeneity, and also could be utilized as the bedsides monitoring tool to continuously evaluate sepsis. The further investigation will develop the related kits for rapid detection the screened molecules, pathways and immune cells.

For rapidly estimate prognosis of septic patients, the previous prediction models and classical biomarkers could not precisely identify severe patients in external dataset [32-36]. The current Molecule-risk model performed well in one external cohort with C-index and AUC as 0.790 and 0.810 . To furthermore validate the novel value of the current model, the current study compared our models with the traditional indicators including procalcitonin (PCT) and interleukin-8 (IL-8) in discriminative ability via receiver operating characteristic curves, shown in Fig. 6. The results indicated that the model performances of the Molecule-risk model and other models were obviously superior to the traditional indicators in clinical value. Since the patients with high risk scores calculated by molecular model were very sick sepsis, the recommended treatments for could be

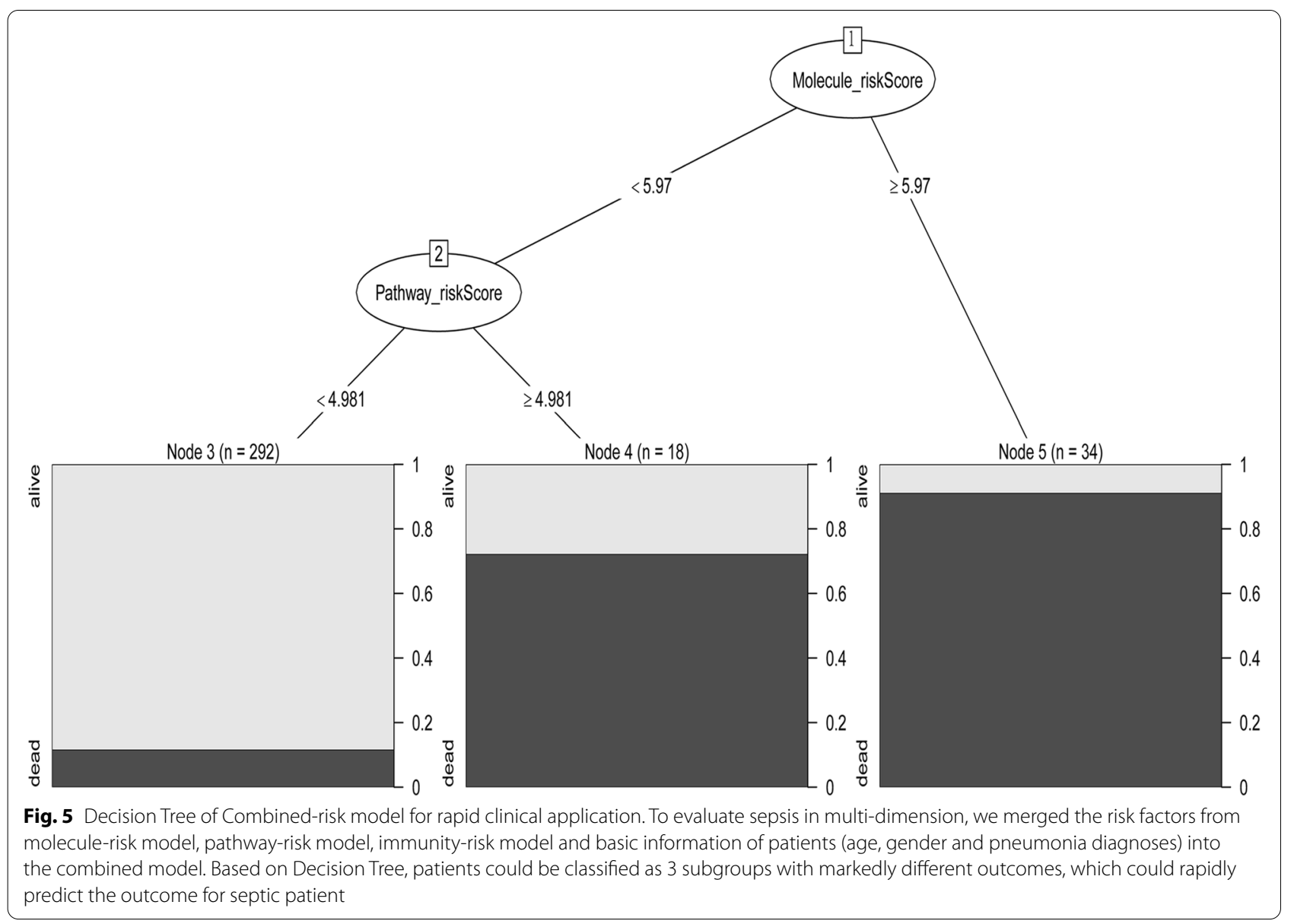


Table 2 The therapeutic recommendations for patients with high risk score

\begin{tabular}{llc}
\hline Models & Therapeutic measures for patients with high risk score & $\begin{array}{c}\text { Number } \\
\text { of } \\
\text { features }\end{array}$ \\
\hline Molecule-risk & Be diverted to intensive care unit for maximal intervention & 8 \\
Pathway-risk & 1.To improve oxygen delivery and decrease oxygen consumption; & 9 \\
Immunity-risk & 2. More hemodynamic measurement to guide therapy. & 4 \\
& 1. To promote immunity therapy such as interferon and thymosin; & 2. To be on guard against superinfection occurring.
\end{tabular}

In Table 2, we provided the therapeutic recommendations for patients with high risk score identified by three sub-models. In addition, the numbers of features in each sub-model were also shown in Table 2

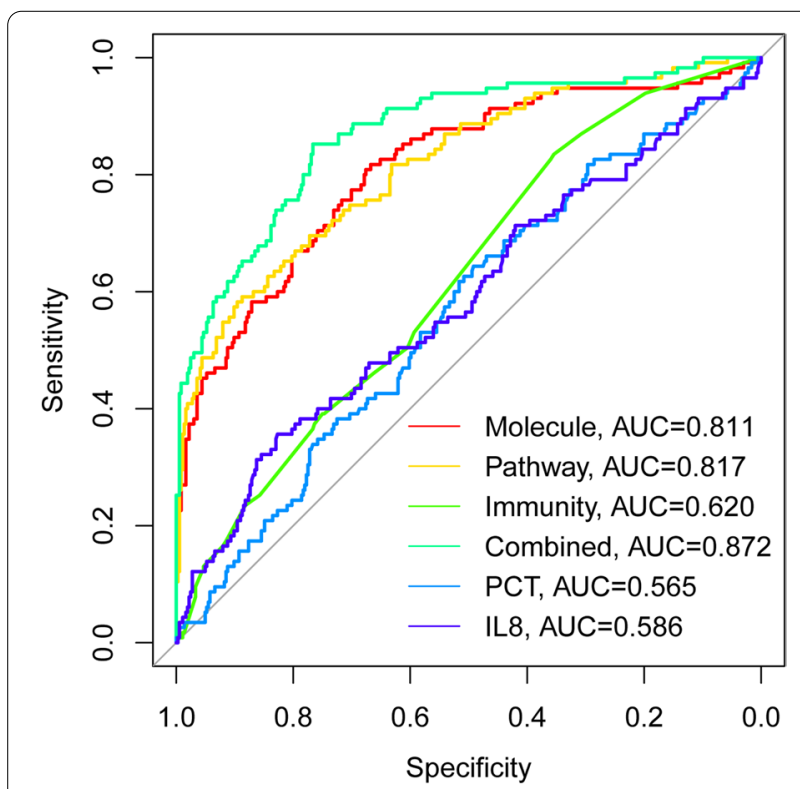

Fig. 6 Model evaluation on 3 sub-models, Combined-risk model and traditional indictors (PCT and IL8) in external validation cohort. The ROC indicated that the AUC of Molecule-risk model, Pathway-risk model, Immunity-risk model, Combined-risk model, IL8 and PCT were $0.810,0.818,0.621,0.873,0.585$ and 0.565

transferred to ICU for more intensive monitor and treatments, while patients predicted to own favorable prognosis were watched in the general wards safely.

For identifying the heterogeneous pathogenesis in sepsis, previous studies [9, 35-37] for risk stratification including clinical severity scores such as Acute Physiology and Chronic Health Evaluation (APACHE) or Sequential Organ Failure Assessment (SOFA) as well as molecular biomarkers could not categorize sepsis at pathogenesis level. It is well known that even in patients with similar clinical features, underlying pathophysiological mechanisms such as signal transduction pathway are significant different. The current pathway-risk model intrinsically uncovered a part of heterogeneous pathogenesis for further selection of specific therapy.
The Pathway-risk score showed that signal transduction pathways of hypoxia, cellular injury and negative feedback repair, metabolic disorder and immune dysregulation were significantly associated with adverse outcomes of sepsis (Fig. 3). These data demonstrate several pathways in sepsis consistent with current knowledge of the pathology of this disease [38-45]. In addition, pathwayrisk score uncovered biological pathway activity variation for accurate identification of high risk patients at pathogenesis level. Compared with surface appearances such as lactic acid, hypoxia inducible factor-1 pathway was classically recognized as intrinsic and robust indictor of tissue perfusion insufficiency. Similarly, steroid biosynthesis pathway, nitrogen metabolism pathway and liver kinase B1- AMP-activated protein kinase pathway could identify heterogeneity of metabolic disorder for sepsis. Simultaneously, mitochondrial membrane damage-related pathway (activating transcription factor-2 pathway), apoptosis-related pathway (telomeres, telomerase, cellular aging, and immortality pathway) and negative feedback repair-related pathway (GRB2 associated binding protein 1 signalosome and transforming growth factor-beta receptor signaling activates SMADs) could uncover some heterogeneity of cellular injury for sepsis. As patients with high risk scores calculated by pathway model could have hypoxia and lower organ perfusion, the current study recommended to provide more hemodynamic measurement, improve oxygen delivery and decrease oxygen consumption for these patients.

For classifying immune status in sepsis, conventional immunity-model $[46,47]$ built by inflammatory markers could not uncover immune cells dysfunction or/and dyshomeostasis, the immunity risk score from current nomograms could predict immune suppressed states based on immune cell subsets. Consistent with previous studies of immunity of sepsis, the Immunity-risk score demonstrated that states of prolonged immune dysfunction leading to immune paralysis are more detrimental to sepsis survival [15]. Immune paralysis is characterized that naïve immune cells (M0 macrophages, B cells naïve and 
$\mathrm{T}$ cells naïve) are hardly activated into mature inflammatory cells, accompanying with an absolutely increasing proportion of immunosuppressive cells such as M2 macrophages and Treg [15, 19, 36, 37]. Consequently, the patients with high Immunity-risk score should be alert of superinfection due to states of immune suppression. Subsequently, promoted immunity such as interferon or thymosin and avoidance of probable infection such as removing inessential catheters could be considered possibly. On the contrary, as a therapy for improving cardiovascular responses to diverse stress stimulation, corticosteroids could be safely used for patients with low Immunity-risk score.

In addition, for rapid clinical application, Decision Tree algorithm of Combined-risk model was utilized to furthermore simplify the model, which could assist clinicians conveniently and efficiently [29].

The value of GSVA algorithm is a classical non-parametric and unsupervised functional enrichment analysis $[17,18]$. Because GSVA could not depend on interest gene subset such as differential genes but on the whole ranking of genes, GSVA has been demonstrated to provide better sensitivity to screen gene expression variations of tiny magnitude which perform coordinately in signal transduction pathway related genes [40, 41]. Conventional ways to investigate immune cell disorders, such as immunohistochemistry and flow cytometry, depend on limited phenotypic markers, and sample disaggregation prior to experiments result in lost or damaged cells, which could change the conclusions of flow cytometry. CIBERSORT outperformed other ways for cell type identification of tissue and blood due to this computational approach reference to gene expression profiles of specific cell rather than one or two markers [19-21].

Overfitting problems unavoidably encountered in prediction models, such as inflation of regression coefficients with while deflation of standard errors, which ultimately reducing both the parsimony of the model and the generalizability of conclusions. Lasso algorithm was more effective for these problems especially in high-dimensional data such as gene expression profiles owing to stringency of lasso penalties. In addition, both backward and forward stepwises selection with AIC could further reduced authentic predictor variables into the final model, and simultaneously preserved model performance.

\section{Conclusions}

As a secondary analysis of publicly available data, detailed information such as the severity, complications, and individual treatment of each patient could not be downloaded for further analysis. In addition, despite the present combined risk model performing well on one external validation, progressive research should be conducted in the future.

\section{Abbreviations}

GEO: Gene Expression Omnibus; AUC: The area under the receiver operating characteristic curve; PCT: Procalcitonin; IL-8: Interleukin-8; SM: Supplemental Material; GSVA: Gene set variation analysis; CIBERSORT: Cell type identification by estimating relative subset of known RNA transcripts; IQR: Low interquartile ranges; FDR: False discovery rate; LASSO: Least absolute shrinkage and selection operator; AIC: Akaike information criterion; HR: Hazard ratio; APACHE: Acute Physiology and Chronic Health Evaluation; SOFA: Sequential Organ Failure Assessment.

\section{Supplementary Information}

The online version contains supplementary material available at https://doi. org/10.1186/s12871-021-01552-x.

Additional file 1.

Additional file 2.

Additional file 3.

\section{Acknowledgements}

We deeply appreciate the data contributors of this study. In addition, we are grateful the reviewers' kind suggestion to furthermore improve this study.

\section{Authors' contributions}

SZ took responsibility for the integrity and the accuracy of the data analysis. ZX-Y and SZ wrote this manuscript. ZX-Y, CY-X, GH-L, LB-P, JW, JP and RZ contributed to the revision of this paper. The authors read and approved the final manuscript.

\section{Funding}

Supported in part by grants from Scientific Research Start-up Funds for talent introduction of Jinan Central hospital (grant numbers: YJRC2021010).

Availability of data and materials

The original data were downloaded from the public database-GEO database (https://www.ncbi.nlm.nih.gov/geo/). The GSE number was GSE63042 and GSE65682.

\section{Declarations}

\section{Ethics approval and consent to participate}

The present study was not required ethics approval since it is a secondary bioinformatics analysis based on public database (GEO database).

\section{Consent for publication}

Not Applicable.

\section{Competing interests}

tablThe authors declare that they have no known competing financial interests or personal relationships that could have appeared to influence the work reported in this paper.

\section{Author details}

${ }^{1}$ Department of Pulmonary and Critical Care Medicine, Jinan Central Hospital Affiliated to Shandong First Medical University and Shandong Academy of Medical Sciences, Cheeloo College of Medicine, Shandong University, No. 105 Jiefang Road, Jinan 250013, Shandong Province, China. ${ }^{2}$ Department of Pulmonary and Critical Care Medicine, Jinan Central Hospital Affiliated to Shandong First Medical University, Jinan, China.

Received: 16 June 2021 Accepted: 21 December 2021

Published online: 07 January 2022 


\section{References}

1. Seymour CW, Liu VX, Iwashyna TJ, Brunkhorst FM, Rea TD, Scherag A, et al. Assessment of clinical criteria for sepsis: for the third international consensus definitions for sepsis and septic shock (Sepsis-3). JAMA. 2016:315:762-74

2. Reinhart K, Daniels R, Kissoon N, Machado FR, Schachter RD, Finfer S, et al. Recognizing sepsis as a global health priority - a WHO resolution. N Engl J Med. 2017;377:414-7.

3. Fleischmann C, Scherag A, Adhikari NK, Hartog CS, Tsaganos T, Schlattmann P, et al. Assessment of global incidence and mortality of hospital-treated sepsis. Current estimates and limitations. Am J Respir Crit Care Med. 2016;193:259-72.

4. Sweeney TE, Perumal TM, Henao R, Nichols M, Howrylak JA, Choi AM, et al. A community approach to mortality prediction in sepsis via gene expression analysis. Nat Commun. 2018;9:694

5. Seymour CW, Kennedy JN, Wang S, Chang CH, Elliott CF, Xu Z, et al. Derivation, validation, and potential treatment implications of novel clinical phenotypes for sepsis. JAMA. 2019;321(20):2003-17.

6. Fang F, Zhang Y, Tang J, Lunsford LD, Li T, Tang R, et al. Association of corticosteroid treatment with outcomes in adult patients with sepsis: a systematic review and meta-analysis. JAMA Intern Med. 2019;179:213-23.

7. Arens C, Bajwa SA, Koch C, Siegler BH, Schneck E, Hecker A, et al. Sepsisinduced long-term immune paralysis--results of a descriptive, explorative study. Crit Care. 2016;20:93

8. Delano MJ, Ward PA. Sepsis-induced immune dysfunction: can immune therapies reduce mortality? J Clin Invest. 2016;126:23-31.

9. Scicluna BP, van Vught LA, Zwinderman AH, Wiewel MA, Davenport EE, Burnham KL, et al. Classification of patients with sepsis according to blood genomic endotype: a prospective cohort study. Lancet Respir Med. 2017:5:816-26.

10. van der Poll T, van de Veerdonk FL, Scicluna BP, Netea MG. The immunopathology of sepsis and potential therapeutic targets. Nat Rev Immunol. 2017;17:407-20.

11. Davenport EE, Burnham KL, Radhakrishnan J, Humburg P, Hutton $P$, Mills TC, et al. Genomic landscape of the individual host response and outcomes in sepsis: a prospective cohort study. Lancet Respir Med. 2016:4:259-71.

12. Blalock WL, Weinstein-Oppenheimer C, Chang F, Hoyle PE, Wang XY, Algate PA, et al. Signal transduction, cell cycle regulatory, and anti-apoptotic pathways regulated by IL-3 in hematopoietic cells: possible sites for intervention with anti-neoplastic drugs. Leukemia. 1999:13:1109-66.

13. Yu S, Huang S, Ding Y, Wang W, Wang A, Lu Y. Transient receptor potential ion-channel subfamily $\vee$ member 4 : a potential target for cancer treatment. Cell Death Dis. 2019;10:497.

14. Escors D, Gato-Cañas M, Zuazo M, Arasanz H, García-Granda MJ, Vera R, et al. The intracellular signalosome of PD-L1 in cancer cells. Signal Transduct Target Ther. 2018;3:26.

15. Schetter AJ, Leung SY, Sohn JJ, et al. MicroRNA expression profiles associated with prognosis and therapeutic outcome in colon adenocarcinoma. JAMA. 2008;299(4):425-36.

16. Silvestri GA, Vachani A, Whitney D, et al. A bronchial genomic classifier for the diagnostic evaluation of lung Cancer. N Engl J Med. 2015;373(3):243-51.

17. Mikacenic C, Price BL, Harju-Baker S, O'Mahony DS, Robinson-Cohen C, Radella F, et al. A two-biomarker model predicts mortality in the critically ill with sepsis. Am J Respir Crit Care Med. 2017;196:1004-11.

18. Hanzelmann S, Castelo R, Guinney J. GSVA: gene set variation analysis for microarray and RNA-seq data. BMC Bioinformatics. 2013;14:7.

19. Newman AM, Liu CL, Green MR, Gentles AJ, Feng W, Xu Y, et al. Robust enumeration of cell subsets from tissue expression profiles. Nat Methods. 2015;12:453-7.

20. Chen B, Khodadoust MS, Liu CL, Newman AM, Alizadeh AA. Profiling tumor infiltrating immune cells with CIBERSORT. Methods Mol Biol. 2018;1711:243-59.

21. Gentles AJ, Newman AM, Liu CL, Bratman SV, Feng W, Kim D, et al. The prognostic landscape of genes and infiltrating immune cells across human cancers. Nat Med. 2015;21:938-45.

22. Zhang S, Wu Z, Xie J, Yang Y, Wang L, Qiu H. DNA methylation exploration for ARDS: a multi-omics and multi-microarray interrelated analysis. J Transl Med. 2019:17(1):345.
23. Zhang S, Liu F, Wu Z, Xie J, Yang Y, Qiu H. Contribution of m6A subtype classification on heterogeneity of sepsis. Ann Transl Med. 2020;8(6):306.

24. Zhang S, Wu Z, Chang W, Liu F, Xie J, Yang Y, et al. Classification of patients with Sepsis according to immune cell characteristics: a Bioinformatic analysis of two cohort studies. Front Med (Lausanne). 2020;3(7):598652.

25. González-Recio O, de Maturana EL, Vega AT, Engelman CD, Broman KW. Detecting single-nucleotide polymorphism by single-nucleotide polymorphism interactions in rheumatoid arthritis using a two-step approach with machine learning and a Bayesian threshold least absolute shrinkage and selection operator (LASSO) model. BMC Proc. 2009;3(Suppl 7):S63.

26. Marami Milani MR, Hense A, Rahmani E, Ploeger A. Applying least absolute shrinkage selection operator and akaike information criterion analysis to find the best multiple linear regression models between climate indices and components of cow's milk. Foods. 2016;5(3):52.

27. Tsalik EL, Langley RJ, Dinwiddie DL, Miller NA, Yoo B, van Velkinburgh JC, et al. An integrated transcriptome and expressed variant analysis of sepsis survival and death. Genome Med. 2014;6(11):111.

28. Zhang $S$, Jing $Y$, Zhang $M$, Zhang $Z$, Ma P, Peng H, et al. Stroma-associated master regulators of molecular subtypes predict patient prognosis in ovarian cancer. Sci Rep. 2015:5:16066.

29. Mortazavi H, Safi Y, Baharvand M, Rahmani S, Jafari S. Peripheral Exophytic Oral lesions: a clinical decision tree. Int J Dent. 2017;2017:9193831.

30. Levy MM, Fink MP, Marshall JC, et al. 2001 SCCM/ESICM/ACCP/ ATS/SIS international sepsis definitions conference. Crit Care Med. 2003:31:1250-6.

31. Calandra T, Cohen J. The international sepsis forum consensus conference on definitions of infection in the intensive care unit. Crit Care Med. 2005;33:1538-48.

32. Shankar-Hari M, Phillips GS, Levy ML, Seymour CW, Liu VX, Deutschman CS, et al. Developing a new definition and assessing new clinical criteria for septic shock: for the third international consensus definitions for sepsis and septic shock (Sepsis-3). JAMA. 2016;315:775-87.

33. Reddy B Jr, Hassan U, Seymour C, Angus DC, Isbell TS, White K, et al. Point-of-care sensors for the management of sepsis. Nat Biomed Eng. 2018:2:640-8

34. Giannakopoulos K, Hoffmann U, Ansari U, Bertsch T, Borggrefe M, Akin I, et al. The use of biomarkers in sepsis: a systematic review. Curr Pharm Biotechnol. 2017;18:499-507.

35. Leligdowicz A, Matthay MA. Heterogeneity in sepsis: new biological evidence with clinical applications. Crit Care. 2019;23:80.

36. Singer M. Sepsis: personalization v protocolization? Crit Care. 2019;23:127

37. Oda S, Hirasawa H, Sugai T, Shiga H, Nakanishi K, Kitamura N, et al. Comparison of Sepsis-related organ failure assessment (SOFA) score and CIS (cellular injury score) for scoring of severity for patients with multiple organ dysfunction syndrome (MODS). Intensive Care Med. 2000;26:1786-93.

38. Escuin-Ordinas H, Li S, Xie MW, Sun L, Hugo W, Huang RR, et al. Cutaneous wound healing through paradoxical MAPK activation by BRAF inhibitors. Nat Commun. 2016:7:12348

39. Nguyen CB, Kumar S, Zucknick M, Kristensen VN, Gjerstad J, Nilsen H, et al Associations between clinical symptoms, plasma norepinephrine and deregulated immune gene networks in subgroups of adolescent with chronic fatigue syndrome. Brain Behav Immun. 2019:76:82-96.

40. Bertocci MA, Bebko G, Versace A, Fournier JC, lyengar S, Olino T, et al. Predicting clinical outcome from reward circuitry function and white matter structure in behaviorally and emotionally dysregulated youth. Mol Psychiatry. 2016;21:1194-201.

41. Hotchkiss RS, Karl IE. Reevaluation of the role of cellular hypoxia and bioenergetic failure in sepsis. JAMA. 1992;267:1503-10.

42. Peyssonnaux C, Cejudo-Martin P, Doedens A, Zinkernagel AS, Johnson RS, Nizet V. Cutting edge: essential role of hypoxia inducible factor1alpha in development of lipopolysaccharide-induced sepsis. J Immunol. 2007;178:7516-9.

43. Cheng SC, Scicluna BP, Arts RJ, Gresnigt MS, Lachmandas E, GiamarellosBourboulis EJ, et al. Broad defects in the energy metabolism of leukocytes underlie immunoparalysis in sepsis. Nat Immunol. 2016;17:406-12.

44. Alexander A, Walker CL. The role of LKB1 and AMPK in cellular responses to stress and damage. FEBS Lett. 2011;585(7):952-7.

45. Hu BR, Fux CM, Martone ME, Zivin JA, Ellisman MH. Persistent phosphorylation of cyclic AMP responsive element-binding protein and activating 
transcription factor-2 transcription factors following transient cerebral ischemia in rat brain. Neuroscience. 1999;89(2):437-52.

46. Chiu CP, Harley CB. Replicative senescence and cell immortality: the role of telomeres and telomerase. Proc Soc Exp Biol Med. 1997;214(2):99-106.

47. Heidecke CD, Weighardt $\mathrm{H}$, Hensler T, Bartels $\mathrm{H}$, Holzmann B. Immune paralysis of $\mathrm{T}$ cells and monocytes during postoperative abdominal sepsis. Correlation of immune functions with outcome. Chirurg. 2000;71(2):159-65.

\section{Publisher's Note}

Springer Nature remains neutral with regard to jurisdictional claims in published maps and institutional affiliations.

- fast, convenient online submission

- thorough peer review by experienced researchers in your field

- rapid publication on acceptance

- support for research data, including large and complex data types

- gold Open Access which fosters wider collaboration and increased citations

- maximum visibility for your research: over $100 \mathrm{M}$ website views per year

At $\mathrm{BMC}$, research is always in progress.

Learn more biomedcentral.com/submissions 